\title{
P-REPRESENTABLE OPERATORS IN BANACH SPACES
}

\section{ROSHDI KHALIL}

Department of Mathemiatics

The University of Michigan

Ann Arbor, Michigan, 48109, U.S.A.

(Received November 7, 1985)

ABSTRACT. Let $E$ and $F$ be Banach spaces. An operator $T \in L(E, F)$ is called $p$-representable if there exists a finite measure $\mu$ on the unit ball, $B\left(E^{\star}\right)$, of $E^{\star}$ and a function $g \in L^{q}(u, F), \frac{1}{p}+\frac{1}{q}=1$, such that

$$
T x=\int_{B\left(E^{\star}\right)}<x, x^{\star}>g\left(x^{\star}\right) d_{i}\left(x^{\star}\right)
$$

for all $x \in E$. The object of this paper is to investigate the class of all $p$-representable operators. In particular, it is shown that $p$-representable operators form a Banach ideal which is stable under injective tensor product. A characterization via factorization through $L^{p}$-spaces is given.

KEY WORDS AND PHRASES. Representable Operator, Banach Space, Stable Ideal Operators. 1980 AMS SUBJECT CLASSIFICATION CODE. $47 \mathrm{~B} 10$.

1. INTRODUCTION.

Let $L(E, F)$ be the space of all bounded linear operators from $E$ into $F$, and $B\left(E^{*}\right)$ the unit ball of $E^{\star}$, the dual of $E$. The completion of the injective tensor product of $E$ and $F$ is denoted by $E$ U $F$. Integral operators in $L(E, F)$ were first defined by Grothendieck, [2], as those operators which can be identified with elements in $(E \vee F)^{\star}$. These operators turn to have a nice integral representation. We refer to Jarchow, [4], for statements and proofs of such representations. Later on, Persson and Pietsch, [5], defined p-integral operators in $L(E, F)$ as those operators $T: E \rightarrow F$ such that $\left.T x=i_{B\left(E^{\star}\right)}<x, x^{\star}\right\rangle d f\left(x^{\star}\right)$, for all $x \in E^{\star}$ where $G$ is a vector measure on $B\left(E^{\star}\right)$ with values in $F$ and $\left\|\int_{B\left(E^{\star}\right)} q\left(x^{\star}\right) d G\left(x^{\star}\right)\right\| \leq\left(\int_{B\left(E^{\star}\right)}\left|q\left(x^{\star}\right)\right|^{p} d^{p}\right)^{1 / p}$ for some finite measure $\mu$ on $B\left(E^{\star}\right)$ and all continuous functions $\xi$ on $B\left(E^{\star}\right)$. The representing vector measure for $T$ need not be of bounded variation. Further, if $G$ is of bounded variation and $F$ doesn't have the Radon-Nikodym property, then $T$ need not be a kernel integral operator.

The object of this paper is to study operators which are in some sense kernel ingegral operators. Such operators is a sub-class of Pietsch p-integral operators.

Throughout this paper, if $E$ is a Banach space, then $E^{\star}$ is the dual of $E$ and $B(E)$ the closed unit ball of $E$. If $K$ is a set then $I_{K}$ is the characteristic function of $K$. If $\left(S_{n}, \mu\right)$ is a measure space, then $L^{P}(\Omega, \mu, E)$ is the space of 
all p-Bochner integrable functions defiried on : with values in $E$, for $1 \ldots p$. If $p=x, L^{x}(:,-, E)$ is the space of . Especiali; bounded functions on s. with values in $E$. The reai $1 \leq q \leq x$ always denote the conjugate of $p: \frac{1}{p}+\frac{1}{q}=1$. Most of our terminology and notations are from Pletsch [6] and Dieste 1 and Uhl [1]. We refer to these texts for any notion cited but not defined in this paper.

2. $R_{p}(E, F)$.

DEFINITION 2.1. An operator $T \in L(E, F)$ is called $p$-representable operator if there exists a finite measure $\downarrow$ defined on the Borel sets of $B\left(E^{\star}\right)$ and a function $g: B\left(E^{\star}\right)-F$ such that $\int_{B\left(E^{\star}\right)}\left\|g\left(x^{\star}\right)\right\|^{9} d_{i} \quad{ }^{a}$, and $T x=\sum_{B\left(E^{\star}\right)} \times x, x^{\star}, g\left(x^{\star}\right) d_{\mu}\left(x^{\star}\right)$ for all $x \in E$.

It follows from the definition that every $p$-representable operator is Pietsch-pintegral operator, but not the converse. Let $R_{p}(E, F)$ be the set of all p-representable operators from $E$ into $F$.

LEMMA 2.2. $R_{p}(E, F)$ is a vector space.

PROOF. Let $T_{1}, T_{2} \in R_{p}(E, F)$ such that

$$
T_{i}(x)=\sum_{B\left(E^{\star}\right)}^{i}<x, x^{\star}>g_{j}\left(x^{\star}\right) d_{i}{ }_{j}\left(x^{\star}\right) \text {. }
$$

Set $\mu_{1}=\mu_{1}+i_{2}$. Then $L_{i} \ll L$. Consequently, $d_{\psi_{j}}=f_{j} d_{2}$. Further, since $\mu_{j}(K)<\mu(K)$ for all Borel sets $K$ on $B\left(E^{\star}\right)$, it follows that $0 \leq f_{j}\left(x^{\star}\right) \leq 1$ a.e. $\mu$, $i=1,2$. Let $\tilde{g}\left(x^{\star}\right)=g_{1}\left(x^{\star}\right) f_{1}\left(x^{\star}\right)+g_{2}\left(x^{*}\right) f_{2}\left(x^{\star}\right)$. Since $1 \leq p_{j}<\infty$, and $0 \leq f_{j}\left(x^{\star}\right) \leq l$, we have $\tilde{g} \in L^{q}\left(B\left(E^{\star}\right), i, F\right)$. Further $\left(T_{1}+T_{2}\right)(x)=\int_{B(E \star)}<x, x^{\star}>\dot{g}\left(x^{\star}\right) d u$, for all $x \in E$. This ends the proof.

For $T \in R_{p}(E, F)$, we define

$$
\|T\|_{\sigma(p)}=\inf \left\{\int\left\|g\left(x^{*}\right)\right\|^{q} d u\left(x^{*}\right) !^{1 / q}\right\}
$$
where the infimum is taken over all $g$ and $u$ for which $T(x)=\int_{B(E \star}<x, x^{*}>g\left(x^{\star}\right) d \mu\left(x^{\star}\right)$,
$x \in E$. It is not difficult to show that \|\|$_{G(p)}$ is a norm on $R_{p}(E, F) .\left(E^{*}\right)$

LEMMA 2.3. For $T \in R_{p}(E, F)$, $\|T\| \leq\|T\|_{o}(p)$.

PROOF. Let $T x=\int_{B\left(E^{\star}\right)}\left\langle x, x^{\star}\right\rangle g\left(x^{\star}\right) d \mu\left(x^{\star}\right)$ for some $t$ and $g$ as in the Definition 2.1. Choose $g^{*}$ and $t$ such that $\left(f\left\|g\left(x^{\star}\right)\right\|^{9} d_{u}\left(x^{\star}\right)\right)^{1 / q} \leq\|T\|_{\sigma(p)}+\varepsilon$, for a given small $\varepsilon>0$. Then, using Holder's inequality:

$$
\begin{aligned}
\|T X\| & \leq\left(\int_{B\left(E^{\star}\right)}\left\|g\left(x^{\star}\right)\right\|^{q} d_{u}\left(x^{\star}\right)\right)^{1 / q} \\
& =\| T T_{\sigma}^{\prime \prime}(p)+\varepsilon .
\end{aligned}
$$

Hence $\|T\|=\|T\|_{\sim}(p)+\varepsilon$. Since $\varepsilon$ is arbitrary, the result follows.

LEMMA 2.4. Every element $T \in R_{p}(E, F)$ is an approximable operator in $L(E, F)$.

PROOF. Let $T x=\int_{B\left(E^{\star}\right)}$ - $x, x^{\star} g\left(x^{\star}\right) d_{\cdots} \cdot\left(x^{\star}\right)$, for some finite measure on $B\left(E^{\star}\right)$ and some $g \in L^{q}\left(B\left(E^{\star}\right), \mu, F\right)$. Choose, and $g$ such that 


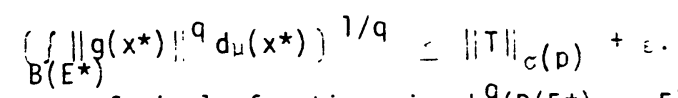

Let $g_{n}$ be a sequence of simple functions in $L^{9}\left(B\left(E^{\star}\right), \ldots, F\right)$ such that $f_{B\left(E^{\star}\right)}\left|! g\left(x^{\star}\right)-g_{n}\left(x^{\star}\right)\right|^{9} d_{u}\left(x^{\star}\right) \rightarrow 0$. Define $T_{n}\left(x^{\star}\right)=f_{Q}\left(E^{\star}\right)^{\star x}, x^{\star}>g_{n}\left(x^{\star}\right) d_{11}\left(x^{\star}\right)$. Then each $T_{n}$ is a finite rank operator, and $\left\|! T-T_{n}\right\|_{\sigma}(p) \rightarrow 0$. Then by definition of approximable operators, Pietsch [6], $T$ is approximable. This ends the proof.

THEOREM 2.5. Let $H, E ; F$ and $G$ be Banach spaces, and $T \in R_{p}(E, F), A \in L(F, G)$ and $B \in L(H, E)$. Then $A T B \in R_{p}(H, G)$ and $\|A T B\|_{\sigma}(p) \leq\|A\|\|B\|\left\|_{i} T\right\|_{\sigma}(p)$

PROOF. Let $T x=\int_{B\left(E^{\star}\right)}<x, x^{\star}>g\left(x^{\star}\right) d_{\mu}\left(x^{\star}\right)$ for all $x \in E$ and some finite measure $\nu$ on $B\left(E^{\star}\right)$ and some $g \in L^{q}\left(B\left(E^{\star}\right), \mu, F\right)$. Then

$$
A T x=\int_{B\left(E^{\star}\right)}<x, x^{\star}>A g\left(x^{\star}\right) d_{u}\left(x^{\star}\right)
$$

and $\int_{B\left(E^{\star}\right)}\left\|A g\left(x^{\star}\right)\right\|^{q} d_{u}\left(x^{\star}\right) \leq\|A\|_{B\left(E^{\star}\right)}\left\|g\left(x^{\star}\right)\right\|^{q} d_{u}\left(x^{\star}\right)$. Hence AT $\in R_{p}(E, G)$ and $\|A T\|_{\sigma}(p) \leq\|A\|_{\|}\|\|_{\sigma}(p) \cdot$

To snow $T B \in R_{p}(H, F)$, let $g_{n}$ be a sequence of simple functions converging to $g$ . in $L^{q}\left(B\left(E^{*}\right), \mu, F\right)$, and $T_{n}$ be the associated operators in $R_{p}(E, F)$. So

$$
T_{n} x=\frac{\int}{B\left(\dot{E}^{\star}\right)}<x, x^{\star}>g_{n}\left(x^{\star}\right) d u\left(x^{\star}\right) \text {. }
$$

With no loss of generality we assume $\|B\|=1$. Define the vector measures $G$ on $B\left(H^{\star}\right)$ into $F$ via:

$$
\begin{aligned}
G_{n}(K) & =\sum_{B^{\prime}\left(E^{\star}\right)} I_{K}\left(y^{\star}\right) d G\left(y^{\star}\right) \\
& =\int_{B\left(E^{\star}\right)} I_{K}\left(B^{\star} x^{\star}\right) S_{n}\left(x^{\star}\right) d_{u}\left(x^{\star}\right) .
\end{aligned}
$$

Clearly, $G_{n}$ is a countably additive vector measure of bounded variation. Further, if we define the measure $"$ on $B\left(H^{\star}\right)$ via

$$
v(K)=\int_{B\left(E^{\star}\right)} T_{K}\left(B^{\star} x^{\star}\right) d \mu\left(x^{\star}\right),
$$

then, using Holder's inequality:

$$
\left.\left\|G_{n}(K)\right\|_{B\left(E^{*}\right)}\left\|g_{n}\left(x^{*}\right)\right\|^{q} d_{i}\left(x^{*}\right) l^{l i q} \cdot[, i K)\right]^{1 / p} .
$$

Hence $G_{n}<v$. Since the range of $G_{n}$ is finite dimensional, it has the Radon-Nikodym property, and consequently there exists $S_{n} \in L^{\prime}\left(B^{\prime}\left(H^{*}\right)\right.$, ,F) such that $d G_{n}=S_{n} d_{v}$.

Further, it is easy to chech that $S_{n} \in L^{q}\left(B\left(H^{*}\right), \ldots, F\right)$.

An application of the Hahn-Banach theorem, we get:

$$
\begin{aligned}
& T_{n} B y=\underset{B\left(E^{*}\right)}{i} B y, x^{*} \cdot g_{n}\left(x^{\star}\right) d_{-}\left(x^{*}\right) \\
& =\underset{B\left(H^{\star}\right)}{\vdots} \cdot y, y^{\star} \cdot S_{n}\left(y^{\star}\right) d:\left(y^{\star}\right) .
\end{aligned}
$$

Since the function $B y, x^{\star}$. is nounded on $B\left(E^{\star}\right)$, the sequence $\left(. B v, \cdot>g_{n}\right)$ is Cauchy in $L^{q}\left(B\left(E^{*}\right), \ldots, F\right)$. Consequentily the sequence $\left(y, \cdot S_{n}\right)$ is Cauchy in $L^{q}\left(B\left(H^{*}\right), i, F\right)$. Let $y, \cdot S$ be the limit of $\left(\cdot y, \cdots S_{n}\right)$ in $L^{q}\left(B\left(H^{\star}\right), \ldots, F\right)$. It is not difficult to see that $T_{n}{ }^{G}$ converges in the operator norm to the operator $J_{y}=C_{B\left(H^{\star}\right)}^{1}, y, y^{\star}, S^{\prime}\left(y^{\star}\right) d\left(y^{\star}\right)$. 
However $T_{n} B-T B$ in the opserator normi. Hence $T B y=j_{B\left(H^{\star}\right)}-y, y^{\star}, S\left(y^{\star}\right) a_{u}\left(y^{\star}\right)$, and $T B \in R_{2}(H, F)$. Further $: T B=-U T T_{0} \| B !$. This enas the proof.

Theorem 2.5 states that $\left(R_{p}, i_{g}, i_{g}\right)$ is a normed operator ideal, $[6]$.

DEFINITION 2.6. Let $\left(.,,_{-}\right)$be a measure space and $F$ a Banach space. An operator $T \in L\left(L^{P}(\delta, \mu), F\right)$ is called $B$-vecior integral operator if there exists $g \in L^{q}(s, \mu, F)$ such inat

for all $f \in L^{P}(\delta, \mu)$.

$$
T f=\sum_{:} f(t) g(t) d_{L}(t)
$$

If the function $g$ is only Pettis q-integrable and the integral defining if is the Pettis integral, then $T$ is known to be called vector integral operator [1].

Now using Theorem 2.5 we can prove:

THEOREM 2.7. Let $E, F$ be Banach spaces and $T \in L(E, F)$. The following are equivalent:

(i) $T \in R_{p}(E, F)$

(ii) There exists operators $T_{1} \in L\left(E, L^{P}(\Omega, \mu)\right)$ and $T_{2} \in L\left(L^{P}(\Omega, \mu), F\right)$ for some measure space $(\Omega, \mu)$ such that $T_{2}$ is B-vector integral operator and $T=T_{2} T_{1}$.

PROOF. (i) $\rightarrow(i i)$. Let $T \in R_{p}(E, F)$ and

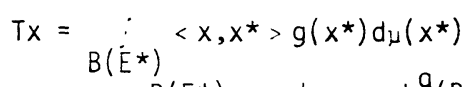

for some finite measure $\mu$ on $B\left(E^{\star}\right)$ and $g \in L^{q}\left(B\left(E^{\star}\right), \mu, F\right)$. Define

and

$$
\begin{gathered}
T_{1}: E \longrightarrow L^{P}\left(B\left(E^{\star}\right)\right) \\
\left(T_{1} x\right)\left(x^{\star}\right)=\left\langle x, x^{\star}\right\rangle,
\end{gathered}
$$

$$
\begin{aligned}
& T_{2}: L^{P}\left(B\left(E^{\star}\right), \mu\right) \longrightarrow F \\
& T_{2}(f)=\int_{B\left(E^{*}\right)} f\left(x^{\star}\right) g\left(x^{\star}\right) d_{\mu} .
\end{aligned}
$$

Then $T_{2}$ is a $B$-vector integral operator and $T=T_{2} T_{1}$.

(ii) $\rightarrow$ (i). Let $T=T_{2} T_{1}, T_{1}\left(E, L^{P}(\Omega, \mu)\right)$ and $T_{2}$ is a B-vector integral operator in $L\left(L^{P}(\Omega, \mu), F\right)$. Then $T_{2} \in R_{p}\left(L^{P}(\Omega, \mu), F\right)$. Using Theorem 2.6, $T_{2} T_{1} \in R_{p}(E, F)$. This ends the proof.

Let $I_{p}(E, F)$ be the space of Pietsch $p$-integral operators from $E$ into $F$, and $\|T\|_{i(p)}$ be the $p$-integral norm for $T \in I_{p}(E, F)$. Clearly $R_{p}(E, F) \subseteq I_{p}(E, F)$ and $\|T\|_{i(p)} \leq\|T\|_{\sigma(p)}$ for all $T \in R_{p}(E, F)$. This, together with the fact that $I_{p}(E, F)$ is complete, [5], one can prove:

THEOREM 2.8. $\left(R_{p}(E, F),\|\|_{\sigma(p)}\right)$ is a Banach space.

If $F$ has the Radon Nikodym property, then $R_{1}(E, F)=I_{1}(E, F)$, and by using Corollary 5 in $[1]$, we see that $R_{1}(C(\Omega), F)=I_{1}(E, F)=N_{1}(C(\Omega), F)$, where $N_{1}(E, F)$ is the class of nuclear operators from $E$ into $F$.

Further if $\Gamma_{p}(E, F)$ is the class of $p$-summing operators from $E$ into $F$, then it follows from the Grothendieck-Pietsch represenation theorem $[6]$, that $R_{p}(E, F) \subseteq \pi_{p}(E, F)$ 
3. IDEAL PROPERTIES OF $F_{\text {. }}$.

We let $R_{p}$ denote the oderator ideal of a : p-representable oderators. The following notions are taken from Pietsch [5] and Holuc [3].

(i) An operator ideal $J$ is called regular if for all Banach spaces $E$ and $F$, $T \in J(E, F)$ if and only if $K_{F} T \in J\left(E, F^{\star \star}\right)$, where $K_{F}$ is the natural embedding of $F$ into $F \star \star$.

(ii) $J$ is called closed if the closure of $J(E, F)$ in $L(E, F)$ is $J(E, F)$ for all Banach spaces $E$ and $F$.

(iii) $J$ is called injective if whenever $J_{F} T \in J\left(E, l^{\alpha}\left(B\left(F^{\star}\right)\right)\right.$ ), then $T \in(E, F)$ for all Banach spaces $E$ and $F$. Here $J_{F}$ is the natural embedding of $F$ into $x^{\infty}\left(B\left(F^{\star}\right)\right)$.

(iv) $J$ is called stable with respect to the injective tensor product if $T_{i} \in J\left(E_{i}, F_{i}\right)$, then $T_{1} \otimes T_{2} \in J\left(E_{1} \otimes E_{2}, F_{1} F_{2}\right)$, for all Banach spaces $E_{1}, E_{2}, F_{1}, F_{2}$.

THEOREM 3.1. $R_{p}$ is regular.

PRO0F. Let $E$ and $F$ be any Banach spaces and let $K_{F} F^{\top} \in R_{p}\left(E, F^{*}\right)$, for $T \in L(E, F)$. Then $K_{F} T x=\int_{B\left(E^{\star}\right)}<x, x^{\star}>g\left(x^{\star}\right) d u^{*}\left(x^{\star}\right)$ for some 2 and $g$ as in Definition 2.l.

Now $g\left(x^{\star}\right) \in K_{F}(F)$ for all $x^{\star} \in B\left(E^{\star}\right)$. Since $K_{F}: F \rightarrow K_{F}(F)$ is an isometric onto operator, the function $g\left(x^{\star}\right)=K_{F}^{-1}\left(g\left(x^{\star}\right)\right)$ is well defined measurable and $\tilde{g} \in L^{q}\left(B\left(E^{\star}\right), \mu, F\right)$. Further

$$
T x=\int_{B\left(\dot{E}^{\star}\right)}<x, x^{\star}>\tilde{g}\left(x^{\star}\right) d_{\mu}\left(x^{\star}\right) .
$$

Hence $T \in R_{p}(E, F)$. This ends the proof.

In a similar way one can prove:

THEOREM 3.2. $R_{p}$ is injective

THEOREM 3.3. $R_{p}$ is stable.

PROOF. Let $T_{i} \in R_{p}\left(E_{i}, F_{i}\right), i=1,2$ and

$$
\begin{aligned}
& T_{1} x=\int_{B\left(E_{1}^{\star}\right)}^{j}<x, x^{\star}>g_{1}\left(x^{\star}\right) d_{\mu_{1}}\left(x^{\star}\right) \\
& T_{2} x=\int_{B\left(E_{2}^{\star}\right)}<x, x^{\star}>g_{2}\left(x^{\star}\right) d_{\mu_{2}}\left(x^{\star}\right),
\end{aligned}
$$

where $\mu_{j}$ and $g_{i}$ be the associated measures and functions as in Definition 2.1. If $E_{i} \delta F_{i}, \quad i=1,2$, is the completion of the injective tensor product of $E_{i}$ with $F_{i}$, [1], then $T_{1} \otimes T_{2} \in L\left(E_{1} \otimes F_{1}, E_{2} \otimes F_{2}\right)$. Further:

$$
\left(T_{1} \otimes T_{2}\right)(x \otimes y)=\int_{B\left(E_{1}^{\star}\right)}\left\langle x, x^{\star}>g_{1}\left(x^{\star}\right) d \mu_{1}\left(x^{\star}\right)\right) \int_{B\left(E_{2}^{\star}\right)}<y, y^{\star}>g_{2}\left(y^{\star}\right) d u_{2}\left(y^{\star}\right) .
$$

Let $k$ be the $w^{\star}$-closure of $\left.B\left(E_{1}^{\star}\right) \otimes B\left(E_{2}^{\star}\right)=i x^{\star} \theta y^{\star}: x^{\star} \in B\left(E_{1}^{\star}\right), y^{\star} \in B\left(E_{2}^{\star}\right)\right\}$ in $\left(E_{1} \otimes E_{2}\right)^{\star}$. Since the map $i: E_{1}^{*} \otimes E_{2}^{\star} \longrightarrow E_{1}^{\star} \otimes E_{2}^{\star}$, the projective tensor product of $E_{1}$ with $E_{2}$, is continuous, [7], it follows that the map $r: B\left(E_{1}^{\star}\right) \times B_{2}\left(E_{2}^{\star}\right) \longrightarrow$ $B\left(E_{1}^{\star}\right) \otimes B\left(E_{2}^{\star}\right), \quad \gamma\left(x^{\star}, y^{\star}\right)=x^{\star} \theta y^{\star}$ is continuous. This induces an isometric into 
operator $4: C(K) \longrightarrow C\left(B\left(E_{\eta}^{+}\right), B\left(E_{2}^{*}\right)\right)$ defined by $u(f)=f 0 ;$. Consequentiy, there exists a measure $u$ on $K$ such that

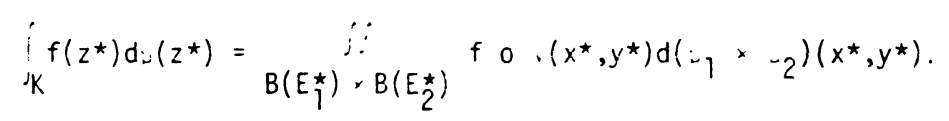

Extend : to $B\left(E_{1} \otimes E_{2}\right)^{\star}$ by putting $: \equiv 0$ on $B\left(E_{1} \otimes E_{2}\right)^{\star} K$. Further define $g: B\left(E_{1} \otimes E_{2}\right)^{\star} \longrightarrow F_{1} \otimes F_{2}$ via $g\left(x^{\star} \otimes y^{\star}\right)=g_{1}\left(x^{\star}\right) \otimes g_{2}\left(y^{\star}\right)$ if $x^{\star} \in B\left(E_{1}^{\star}\right)$, $y^{\star} \in B\left(E_{2}^{\star}\right)$, and $g\left(z^{\star}\right)=0$ otherwise. Then it is not difficult to see that

$$
\left(T, \theta T_{2}\right)(z)=\frac{!}{B\left(E_{1} \otimes E_{2}\right)^{\star}}<z, z^{\star}>g\left(z^{\star}\right) d \mu\left(z^{\star}\right)
$$

for all $z \in E_{1} \otimes E_{2}$. Since $g \in L^{q}\left(B\left(E_{1} \otimes E_{2}\right)^{\star}, \mu, F_{1} \otimes F_{2}\right)$, it follows that $T_{1} \otimes T_{2} \in R_{p}\left(E_{1} \otimes E_{2} \cdot F_{1} \otimes F_{2}\right)$. This ends the proof.

A negative result for $R_{p}$ is the following:

THEOREM 3.4. $R_{p}$ is not closed.

PROOF: Assume $R_{p}$ is closed. Since the ideal of finite rank operator is contained in $R_{p}$, one has the ideal of approximable operators is contained in $R_{p}$. By Lemma 2.4, one gets $R_{p}=$ the ideal of approximable operators. Theorem 2.8, together with the open mapping theorem we get that \|\|$_{\sigma(p)}$ and $\left\|_{1}\right\|$ are equivalent on $R_{p}$. This is a contradiction. Hence $R_{p}$ is not closed.

ACKNOWLEDGEMENT. The author would like to thank Professor Ramanujan and Dr. Defant for stimulating discussions. This work was done while the author was a visiting professor at the University of Michigan. The author would also like to thank the Department of Mathematics at the University of Michigan for their warm hospitality.

\section{REFERENCES}

1. DIESTEL, J. and UHL, J.J. Vector Measures. Mathematical Surveys, 15. Providence, R.I. 1977.

2. GROTHENDIECK, A. Produits Tensoriels Topologiques et Espaces Nucleaires, Mem. Amer. Math. Soc. No. 16, 1955.

3. HOLUB, J.R. Tensor Product Mappings, Math. Ann. 188 (1970) 1-12.

4. JARCHOW, H. Locally Convex Spaces. Teubner Stuttgart, 1981.

5. PERSSON, A. and PIETSCH, A. P-Nucleare und p-Integrable Abbildungen in Banachryumen, Studia Math. 33 (1969) 19-62.

6. PIETSCH, A. Operator Ideal. North Holland Pub. Comp. 1980.

7. SCHAEFER, H. Topological Vector Spaces. New York, Macmillan Co., 1966. 


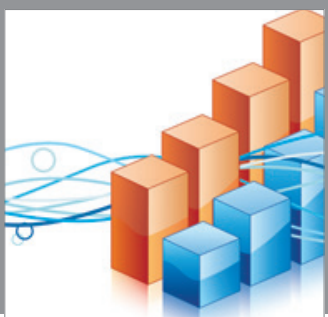

Advances in

Operations Research

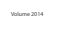

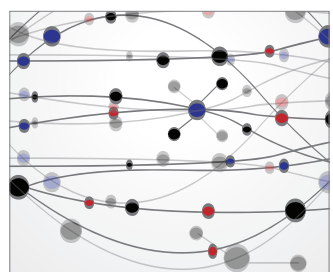

\section{The Scientific} World Journal
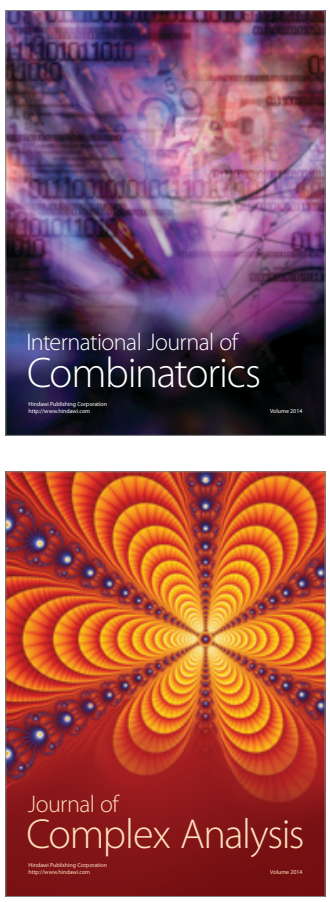

International Journal of

Mathematics and

Mathematical

Sciences
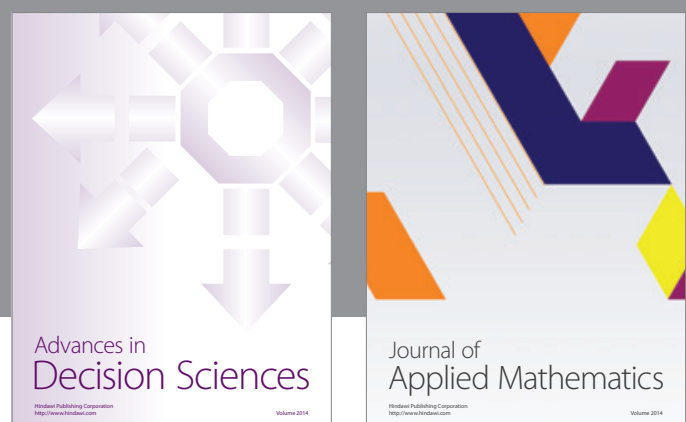

Journal of

Applied Mathematics
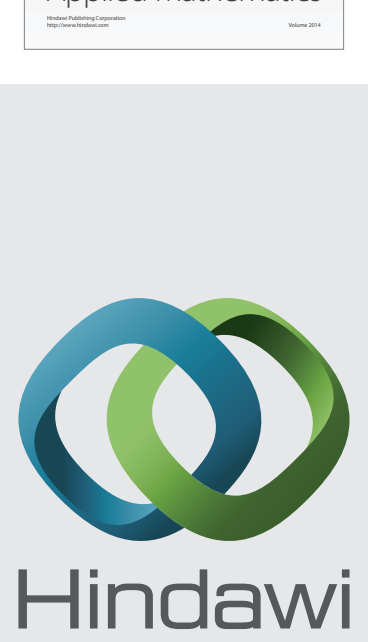

Submit your manuscripts at http://www.hindawi.com
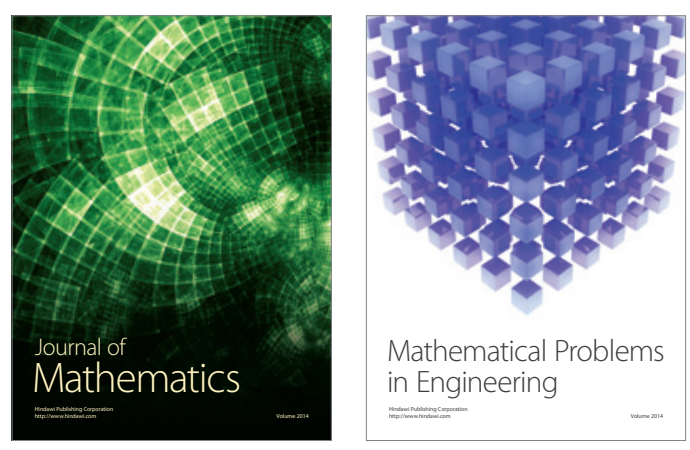

Mathematical Problems in Engineering
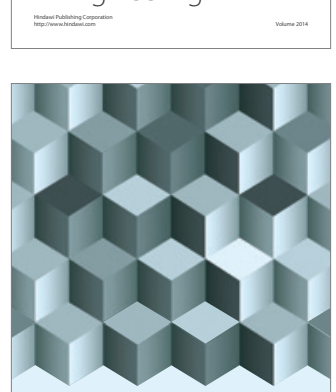

Journal of

Function Spaces
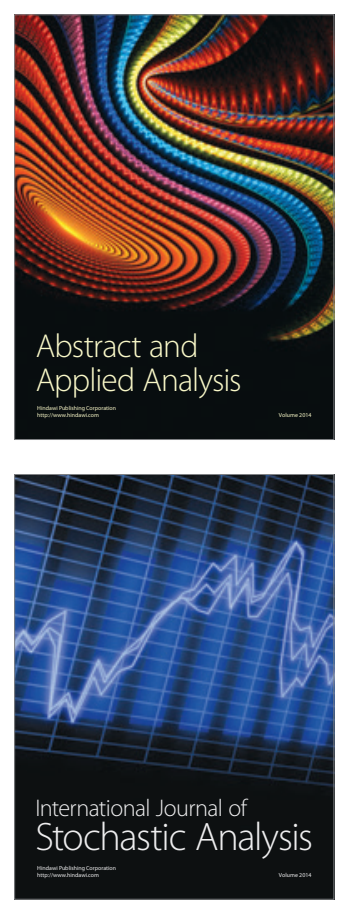

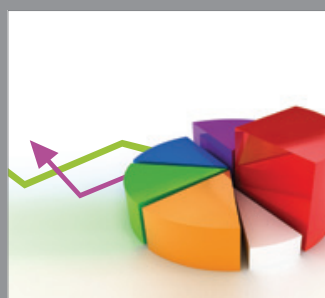

ournal of

Probability and Statistics

Promensencen
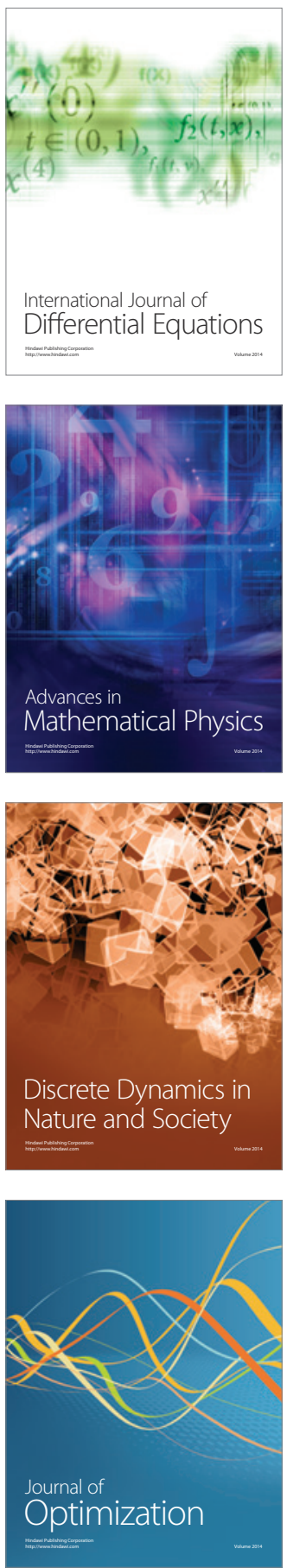